\title{
Downregulation of ASPP1 in gestational trophoblastic disease: correlation with hypermethylation, apoptotic activity and clinical outcome
}

\author{
Victor CY Mak ${ }^{1}$, Lee Lee ${ }^{1}$, Michelle KY Siu ${ }^{1}$, Oscar GW Wong ${ }^{1}$, Xin Lu², Hextan YS Ngan³, \\ Esther SY Wong ${ }^{1}$ and Annie NY Cheung ${ }^{1}$ \\ ${ }^{1}$ Department of Pathology, The University of Hong Kong, Hong Kong, China; ${ }^{2}$ Nutfield Department of Clinical \\ Medicine, Ludwig Institute for Cancer Research, University of Oxford, Oxford, UK and ${ }^{3}$ Department of \\ Obstetrics and Gynaecology, The University of Hong Kong, Hong Kong, China
}

\begin{abstract}
Gestational trophoblastic disease encompasses a spectrum of trophoblastic lesions including true neoplasms such as choriocarcinomas and the potentially malignant hydatidiform moles, which may develop persistent disease requiring chemotherapy. ASPP1, a member of apoptosis-stimulating proteins of p53 (ASPPs), is a proapoptotic protein that can stimulate apoptosis through its interaction with $\mathrm{p} 53$. We evaluated the promoter methylation and expression profiles of ASPP1 in different trophoblastic tissues and its in vitro functional effect on two choriocarcinoma cell lines, namely JEG-3 and JAR. Significant downregulation of ASPP1 mRNA and protein levels was demonstrated in hydatidiform moles and choriocarcinomas, when compared with normal placentas by quantitative-PCR and immunohistochemistry. The ASPP1 mRNA level was significantly correlated with its hypermethylation status, evaluated with methylation-specific PCR, in placenta and gestational trophoblastic disease samples $(P=0.024)$. Moreover, lower ASPP1 immunoreactivity was shown in hydatidiform moles that progressed to persistent gestational trophoblastic neoplasms than in those that regressed $(P=0.045)$. A significant correlation was also found between expression of ASPP1 and proliferative indices (assessed by Ki67 and MCM7), apoptotic activity (M30 CytoDeath antibody), p53 and caspase-8 immunoreactivities. An in vitro study showed that ectopic expression of ASPP1 could trigger apoptosis through intrinsic and extrinsic pathways as indicated by an increase in cleaved caspase-9 and Fas ligand protein expression. The latter suggests a hitherto unreported novel link between ASPP1 and the extrinsic pathway of apoptosis. Our findings suggest that downregulation of ASPP1 by hypermethylation may be involved in the pathogenesis and progress of gestational trophoblastic disease, probably through its effect on apoptosis. Modern Pathology (2011) 24, 522-532; doi:10.1038/modpathol.2010.216; published online 19 November 2010
\end{abstract}

Keywords: apoptotic activity; ASPP1; choriocarcinomas; gestational trophoblastic disease; hypermethylation; methylation

Gestational trophoblastic disease includes partial and complete hydatidiform moles, invasive moles, choriocarcinomas, placenta site trophoblastic tumors and epithelioid trophoblastic tumors. The latter three are frankly invasive neoplasms, whereas

Correspondence: Professor ANY Cheung, MD, FRCPath., Department of Pathology, The University of Hong Kong, Queen Mary Hospital, Pokfulam Road, Hong Kong, China.

E-mail: anycheun@.hkucc.hku.hk

Received 26 May 2010; revised 15 August 2010; accepted 15 August 2010; published online 19 November 2010 hydatidiform moles can be considered as abnormal placentas that are prone to malignant transformation. Most hydatidiform moles regress spontaneously after suction evacuation, whereas $8-30 \%$ will progress to persistent gestational trophoblastic neoplasms that require chemotherapy. The incidence of gestational trophoblastic disease is relatively higher in Asian than in western countries. ${ }^{1}$

Genetic and biological studies are important for enhancing our understanding of the pathogenesis of gestational trophoblastic disease. ${ }^{2}$ From our previous studies, it was evident that gestational 
trophoblastic disease exhibits an increased apoptotic activity when compared with non-molar placentas. Moreover, hydatidiform moles with lower apoptotic activity are associated with the development of gestational trophoblastic neoplasm. ${ }^{3,4}$ The tumor-suppressor protein p53 is an important inducer of apoptosis, ${ }^{5}$ and overexpression of wildtype p53 has been demonstrated in hydatidiform moles and choriocarcinomas when compared with normal placentas. ${ }^{6-9}$ Unlike most other solid tumors, direct cDNA sequencing could not detect any mutation in the overexpressed p53 in gestational trophoblastic disease. ${ }^{6,10}$

Apoptosis-stimulating proteins of p53 (ASPPs) are a recently identified family of proteins, which can regulate the apoptotic function of wild-type p53. It consists of three members, namely ASPP1, ASPP2 and iASPP. $^{11}$ Apoptosis can adopt two distinct pathways-the intrinsic pathway triggered by mitochondrial outer membrane permeabilization and the extrinsic pathway that depends on death signals transduced by cell-surface receptors. Biochemical studies have suggested that ASPP1 and ASPP2 activate apoptosis by stimulating proapoptotic genes such as $B A X$ and PUMA that are involved in the intrinsic pathway. ${ }^{11,12}$ In contrast, iASPP seems to inhibit apoptosis through the competition for p53 binding with ASPP1 and ASPP2. ${ }^{13}$ Owing to their specific ability of stimulating apoptosis without affecting cell-cycle arrest, ${ }^{12}$ ASPP1 and ASPP2 may have a unique role in the p53-mediated apoptotic pathway. Recently, it has been demonstrated that ASPP1 is downregulated in human cancer cell lines expressing wild-type p53, ${ }^{14,15}$ including breast cancer and acute lymphoblastic leukemia. ${ }^{11,16}$ Hypermethylation of gene promoter or the $5^{\prime}$ untranslated region was shown to be a possible epigenetic mechanism that inactivates $A S P P 11^{14,16}$

In this study, we evaluated the expression and methylation profiles of ASPP1 in normal placentas and gestational trophoblastic disease in correlation with clinical parameters, followed by an in vitro functional analysis, in an attempt to unveil the possible role of ASPP1 in the pathogenesis of gestational trophoblastic disease.

\section{Materials and methods}

\section{Clinical Samples and Cell Lines}

For immunohistochemistry, 94 trophoblastic tissue samples, including 18 first-trimester placentas, 12 term placentas, 14 partial moles (gestational age ranged from 8 to 20 weeks, mean =14 weeks), 38 complete moles (gestational age ranged from 5 to 28 weeks, mean $=14$ weeks) and 12 choriocarcinomas were retrieved from the archives of the Department of Pathology (Queen Mary Hospital, The University of Hong Kong). For real-time PCR and methylationspecific PCR (MS-PCR), cDNA and DNA were prepared from snap-frozen samples of 46 hydatidiform moles, 5 choriocarcinomas, 9 term placentas and 17 first-trimester placentas.

First-trimester and term placentas were collected after induced abortion by suction evacuation and normal delivery, respectively. Tissues of hydatidiform moles and choriocarcinomas were obtained from specimens of uterine evacuate and/or hysterectomy. The tissues were collected with the approval of the Institutional Review Board. All tissue sections were histologically reviewed using generally accepted diagnostic criteria.

Gestational trophoblastic neoplasm was diagnosed if there was a plateau in the human chorionic gonadotrophin (hCG) level for 4 weeks or if there was a further increase in hCG for 3 consecutive weeks after evacuation. Most of the hydatidiform moles have been assessed by fluorescent microsatellite genotyping after microdissection and chromosome in situ hybridization. ${ }^{17,18}$ Data on proliferation and apoptosis indices of these cases were also retrieved from previous studies to be correlated with ASPP1 immunoreactivity. ${ }^{3,6,19-21}$

For in vitro studies, two choriocarcinoma cell lines (JEG-3 and JAR) (American Type Culture Collection, Manassas, VA, USA) were cultured in minimum essential Eagle's medium supplemented with $10 \%$ fetal bovine serum and $100 \mathrm{Units} / \mathrm{ml}$ penicillin and streptomycin.

\section{Immunohistochemical Study}

Immunohistochemistry was performed on 5- $\mu \mathrm{m}$ thick deparaffinized sections using UltraVision LP Value Detection System Horseradish Peroxidase Polymer (LabVision, Fremont, CA, USA) ${ }^{22}$ after antigen retrieval by pressure cooking in $1 \mathrm{mM}$ EDTA solution, $\mathrm{pH} 7.6$ for $5 \mathrm{~min}$. A monoclonal mouse anti-human antibody of ASPP1 (LXO54.2) (Sigma, St Louis, MO, USA) was applied in 1:750 dilution and incubated overnight at room temperature. Freshly prepared DAB (3,3'-diaminobenzidine tetrahydrochloride) was applied as a chromogen and sections were counter-stained with hematoxylin. Negative controls were prepared by replacing the primary antibody with PBS. A known positive control from a normal first-trimester placenta was used.

The percentage of immunopositive cells was scored according to the following criteria: 0 , no positivity; $1,0.1-25.0 \%$ of cells immunopositive; 2 , 25.1-50.0\% immunopositive; 3, 50.1-75.0\% immunopositive; and 4, 75.1-100\% of cells immunopositive. A signal intensity score was assigned by the following criteria: 0, immunonegative; 1, weak positivity; 2, moderate positivity; 3, strong positivity; and 4, very strong positivity. Syncytiotrophoblast (ST), a multinucleated cell was counted as one cell. Immunohistochemical scores in a range of 0 , 2-8 were established by adding the percentage of positive scores and signal intensity scores. ${ }^{22,23}$ 
Photomicrographs were taken using Aperio Spectrum (Aperio, Vista, CA, USA).

\section{Quantitative Real-Time PCR}

Quantitative PCR (qPCR) was performed according to previously published procedures..$^{22-24}$ Total RNA $(2.5 \mu \mathrm{g})$ extracted by Trizol reagent (Invitrogen, Life Technologies Inc., Rockville, MD, USA) was subjected to first-strand cDNA synthesis using the SuperScript Reverse Transcriptase system with oligo-dT primers (Invitrogen, Carlsbad, CA, USA). qPCR was performed on the ABI PRISM 7900 Sequence Detection System (Applied Biosystems, Foster City, CA, USA). The cDNA of GAPDH was amplified as the housekeeping control for each sample. Primers used are listed in Table 1. The expression of ASPP1 determined using the $2^{-\Delta \Delta \mathrm{CT}}$ method was normalized with respect to that of GAPDH.

\section{5-aza-dc and TSA Treatment}

Treatments were performed in JEG-3 and JAR cells including a 3-day culture with $5 \mu \mathrm{M}$ 5-aza-2'deoxycytidine (5-aza-dc; Sigma), a 1-day culture with $0.3 \mu \mathrm{M}$ Trichostatin A (TSA; Sigma) and a combination of both $(5 \mu \mathrm{M} 5$-aza-dc for 2 days, followed by $5 \mu \mathrm{M} 5$-aza-dc with $0.3 \mu \mathrm{M}$ TSA for 1 day). A medium with DMSO was used as control. The cultured cells were harvested at indicated time points.

\section{DNA Preparation, Sodium Bisulfite Modification and MS-PCR}

Genomic DNA of $5 \mu \mathrm{g}$ was extracted by phenolchloroform extraction after proteinase $\mathrm{K}$ digestion and subjected to bisulfate treatment for conversion of unmethylated cytosine to uracil as described previously. ${ }^{25,26}$ The QIAEX II kit (Qiagen, Hiden, Germany) was used for subsequent purification of converted DNA.

Methylation- (M) and unmethylation (U)-sensitive primers, as listed in Table 1, were designed to amplify the promoter region $(-681$ to -476$)$ of $A S P P 1$ (GeneBank NM 015316) according to a previous study ${ }^{16}$ for MS-PCR analysis. Universal methylated DNA (Chemicon, Atlanta, GA, USA) and water blanks without template were used as positive and negative controls for each PCR set, respectively. Touchdown PCR was carried out. An initial denaturation at $95{ }^{\circ} \mathrm{C}$ for $10 \mathrm{~min}$ was followed by 5 PCR cycles of $95{ }^{\circ} \mathrm{C}$ for $30 \mathrm{~s}, 59{ }^{\circ} \mathrm{C}$ for $30 \mathrm{~s}$ and $72{ }^{\circ} \mathrm{C}$ for $30 \mathrm{~s}$. The PCR was then completed with 35 cycles of $30 \mathrm{~s}$ at $95{ }^{\circ} \mathrm{C}$ and $30 \mathrm{~s}$ at $55^{\circ} \mathrm{C}$. MSP products were separated on a $2 \%$ agarose gel, stained with ethidium bromide and visualized under UV light.

\section{ASPP1 Transfection, Western Blot Analysis and Subcellular Localization}

JEG-3 and JAR cultures were transfected with the $A S P P 1$ construct (a generous gift from Professor Xin $\mathrm{Lu}$ ) using Lipofectamine 2000 (Invitrogen). The pCDNA 3.1 vector was used as a control. The total protein lysate was extracted with SDS lysis buffer containing proteinase inhibitors. ${ }^{23}$ Protein concentration was determined using a detergent-compatible protein assay (Bio-Rad Laboratories, Hercules, CA, USA). In all, $20 \mu \mathrm{g}$ of protein was resolved by SDS-PAGE and then transferred to polyvinylidene difluoride membrane and probed with corresponding antibodies. The primary antibodies used for western blotting are listed in Table 2. Cytoplasmic and nuclear extracts from JEG-3 were isolated using the ProteoExtract Native Membrane Protein Extraction Kit (Calbiochem, San Diego, CA, USA).

\section{TdT-Mediated dUTP Nick-End Labeling Assay}

TdT-mediated dUTP nick-end labeling assay (TUNEL) was performed using an In Situ Cell Death Detection kit (Roche Biochemical, Indianapolis, IN, USA) as described previously. ${ }^{3}$ The number of TUNEL-positive cells in controls and in JEG-3 and JAR after $A S P P 1$ transient transfection was counted in three different fields at $\times 40$ magnification by fluorescence microscopy.

Table 1 Primers for MS-PCR and RT-PCR studies

\begin{tabular}{lll}
\hline & Forward primer $\left(5^{\prime} \rightarrow 3^{\prime}\right)$ & Reverse primer $\left(5^{\prime} \rightarrow 3^{\prime}\right)$ \\
\hline RT-PCR & & \\
ASPP1 & & \\
GADPH & TTGTCCTCTCATTGCACG & AACTTACCCTCTCAGAGC \\
$F a s$ & TCCATGACAACTTTGGTATCGTG & ACAGTCTTCTGGGTGGCAGTG \\
TRADD & TCAATGAAACTGGGCTGTACTTT & AGAGTCCTCATGTAGACCTTGT \\
TRAF2 & CTCCAAGGGATTGGAATTGA & GACAAAGCCACCCCAAGTTA \\
MS-PCR & TTCTGCGGCTATTGCTGA & TGAAACTGTAAGGGCTGG \\
Methylated allele $(\mathrm{M})$ & CTGACTTGGAGCAGAAGG & GCCGTTCAGGTAGATACG \\
Unmethylated allele $(\mathrm{U})$ & & \\
\hline
\end{tabular}


Table 2 Primary antibodies used in this study

\begin{tabular}{|c|c|c|c|c|c|}
\hline Target protein & Animal source & Catalog no. & Usage & Working dilution & Vendor \\
\hline ASPP1 & Mouse & A4355 & $\begin{array}{l}\text { Immunohistochemistry } \\
\text { and western blot }\end{array}$ & $1: 500$ & Sigma (St Louis, MO, USA) \\
\hline Actin & Rabbit & A5060 & Western blot & $1: 1000$ & Sigma \\
\hline$\alpha$-Tubulin & Mouse & sc-8035 & Western blot & 1:1000 & $\begin{array}{l}\text { Santa Cruz Biotechnology Inc. } \\
\text { (Santa Cruz, CA, USA) }\end{array}$ \\
\hline Histone $\mathrm{H} 1$ & Mouse & sc- 8030 & Western blot & $1: 1000$ & Santa Cruz Biotechnology Inc. \\
\hline Caspase-9 & Rabbit & 9502 & Western blot & $1: 1000$ & Cell Signaling (Beverly, MA, USA) \\
\hline FasL & Rabbit & sc-834 & Western blot & $1: 1000$ & Santa Cruz Biotechnology Inc. \\
\hline Fas & Rabbit & sc-715 & Western blot & 1:1000 & Santa Cruz Biotechnology Inc. \\
\hline
\end{tabular}

\section{Statistical Analysis}

Statistical Package for Social Science (SPSS) version 15.1 was used for statistical analysis including $\chi^{2}$ test for methylation between groups, non-parametric unpaired $t$-test (Mann-Whitney test) for continuous data and Spearman's rho test for correlation analysis. $P$-values $<0.05$ were considered statistically significant.

\section{Results}

\section{ASPP1 was Downregulated in Hydatidiform Moles and Choriocarcinomas and Correlated with Clinical Outcome of Hydatidiform Moles}

In trophoblastic tissues studied, ASPP1 immunoreactivity was predominantly found in the cytoplasm and cell membrane of the villous cytotrophoblasts. Weak and focal staining was also observed in STs, villous mesenchyme, decidua and extravillous intermediate trophoblasts (Figures 1a-e). Significantly decreased immunoreactivity was demonstrated in hydatidiform moles and choriocarcinomas when compared with placentas (Figure 1f). ASPP1 expression was particularly weak in choriocarcinoma with only focal and vague immunoreactivity in occasional ST (Figure 1d). Among hydatidiform moles, complete moles generally demonstrated lower ASPP1 expression than did partial moles, although statistical significance was not reached $(P=0.274)$. More importantly, hydatidiform moles that developed persistent gestational trophoblastic neoplasms had significantly lower ASPP1 expression in cytotrophoblasts compared with those that spontaneously regressed ( $P=0.045$, Mann-Whitney test).

Western blot analysis on subcellular fractions of JEG-3 cells by anti-ASPP1 antibody demonstrated a strong signal at $175 \mathrm{kDa}$ in the cytoplasmic fraction of the protein extract but not in nuclear fractions (Figure 1g). Such findings concurred with the immunohistochemical detection of ASPP1 predominantly in the cytoplasm.

Data retrieval of corresponding cases on proliferation indexes (Ki67 and MCM7) ${ }^{20}$ and apoptotic parameters including apoptosis indices assessed by M30 cytodeath antibody ${ }^{3}$ and p53 expression ${ }^{6}$ was subjected to correlation analysis with ASPP1 expression. Results showed that ASPP1 inversely correlated with p53 (correlation $=-0.482 ; P<0.001$ ), Ki67 (correlation coefficient $=-0.304 ; P=0.020$ ) and MCM7 (coefficient $=-0.409 ; P=0.016$ ) proliferative indexes, as well as with the apoptotic index indicated by M30 (coefficient $=-0.397 ; P=0.004$ ). In addition, the ASPP1 index correlated positively with immunoreactivity of total form of caspase-8 (coefficient $=$ 0.458; $P=0.024) .{ }^{19}$

\section{Methylation Status at the ASPP1 Promoter Region Correlated with mRNA Expression}

Real-time qPCR performed on cDNA samples showed a significantly lower mRNA expression in hydatidiform moles $(P=0.003)$ and choriocarcinomas $(P=0.001)$, than in normal first-trimester placentas (Figure 2a). Choriocarcinomas further showed a significantly lower ASPP1 mRNA expression than did hydatidiform moles $(P=0.002)$. In normal placentas, higher expression was observed in term placentas than in first-trimester placentas $(P=0.006)$.

In vitro studies showed that $A S P P 1$ expression was upregulated by twofold and fivefold by 5 -aza-dc and TSA treatments, respectively, in JEG-3, whereas twofold and sevenfold changes were detected, respectively, in JAR. Double treatment of both drugs increased ASPP1 mRNA expressions by 10- and 5-fold in JEG-3 and JAR, respectively (Figure 2b). These findings implied that DNA methylation is likely to be important in inactivation of ASPP1 in gestational trophoblastic disease.

MS-PCR studies demonstrated a higher frequency of methylated alleles of ASPP1 in hydatidiform moles than that in normal placentas (first-trimester and term placentas) (Figure 2c). Methylated ASPP1 alleles were shown in $34.8 \%(16 / 46)$ cases of hydatidiform moles as compared with $11.8 \%(2 / 17)$ and $22.2 \%(2 / 9)$ in normal first-trimester and term placenta cases, respectively. Furthermore, ASPP1 mRNA expression was significantly correlated with ASPP1 methylation status ( $P=0.029$, Fisher's exact test). Choriocarcinomas possessed both methylated and unmethylated ASPP1 alleles (Figure 2c). 
a

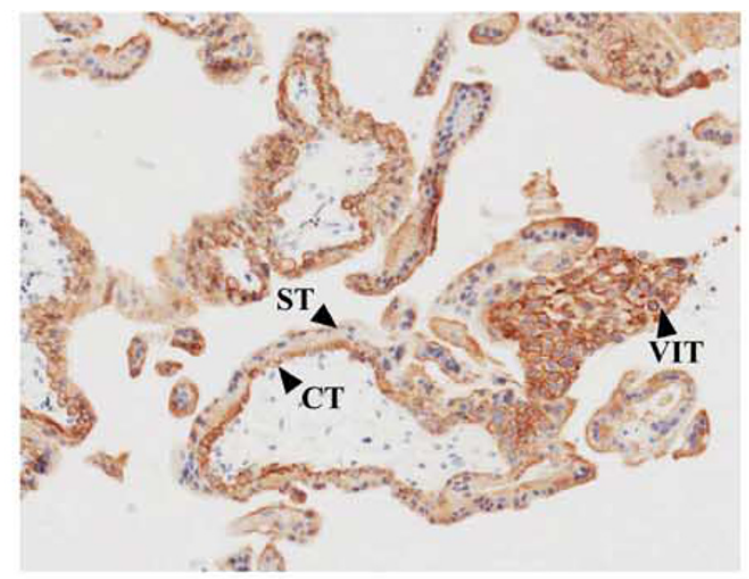

C
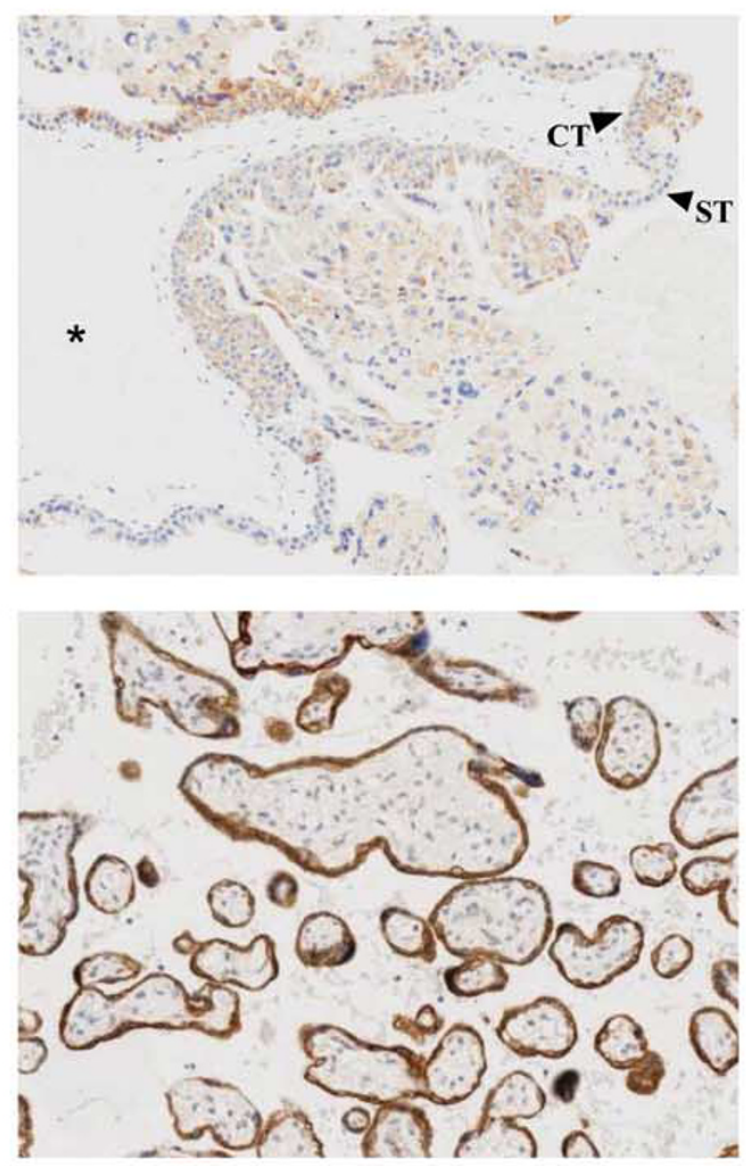

b

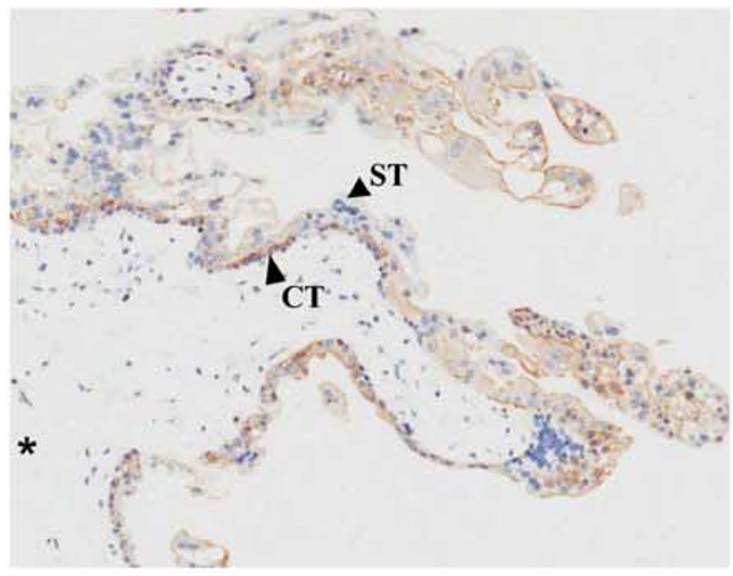

d

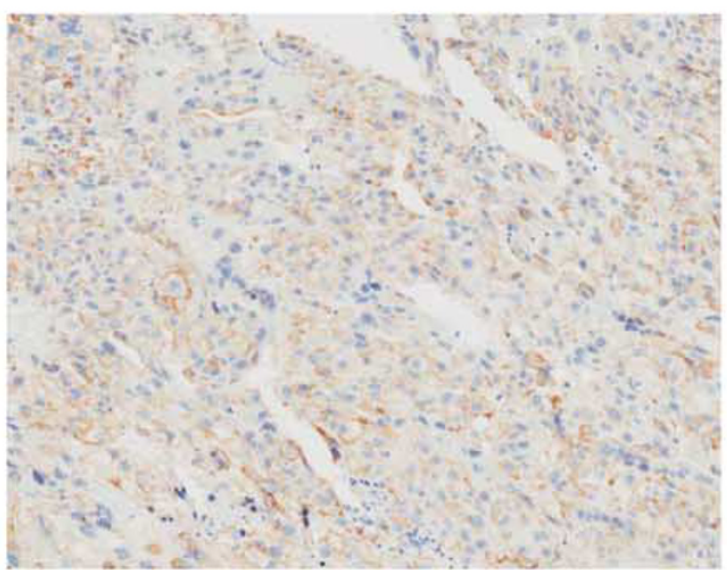

f
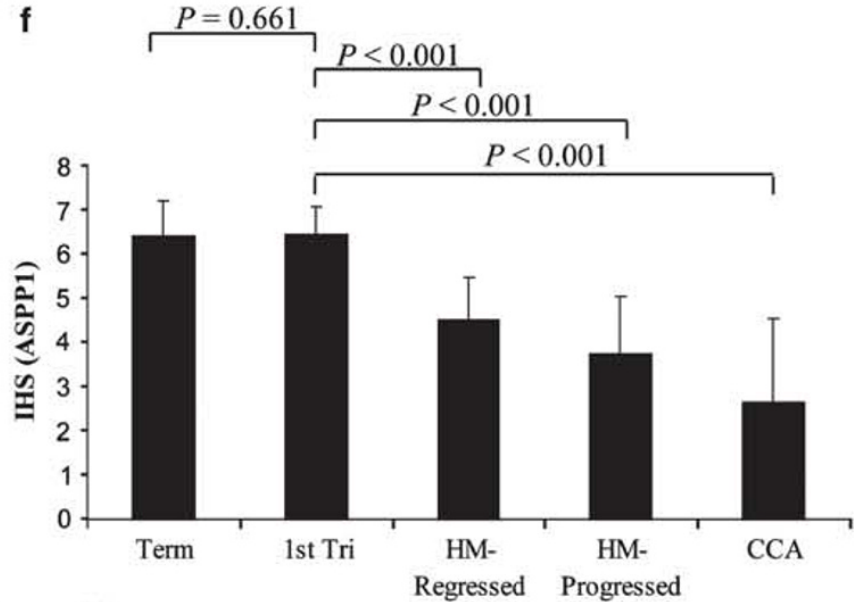

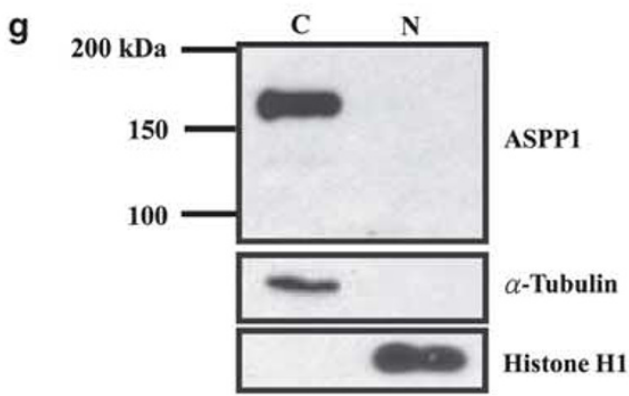




\section{Overexpression of ASPP1 in JEG-3 and JAR Induced Apoptosis and Upregulated FasL Expression}

To investigate the functional role of ASPP1 in apoptosis, transient transfection of $A S P P 1$ was performed in JEG-3 and JAR. Transfection efficiency was assessed $48 \mathrm{~h}$ after transfection by western blot as illustrated in Figure 4. As demonstrated by TUNEL assay, empty vector-transfected JEG-3 and JAR showed a percentage of apoptotic
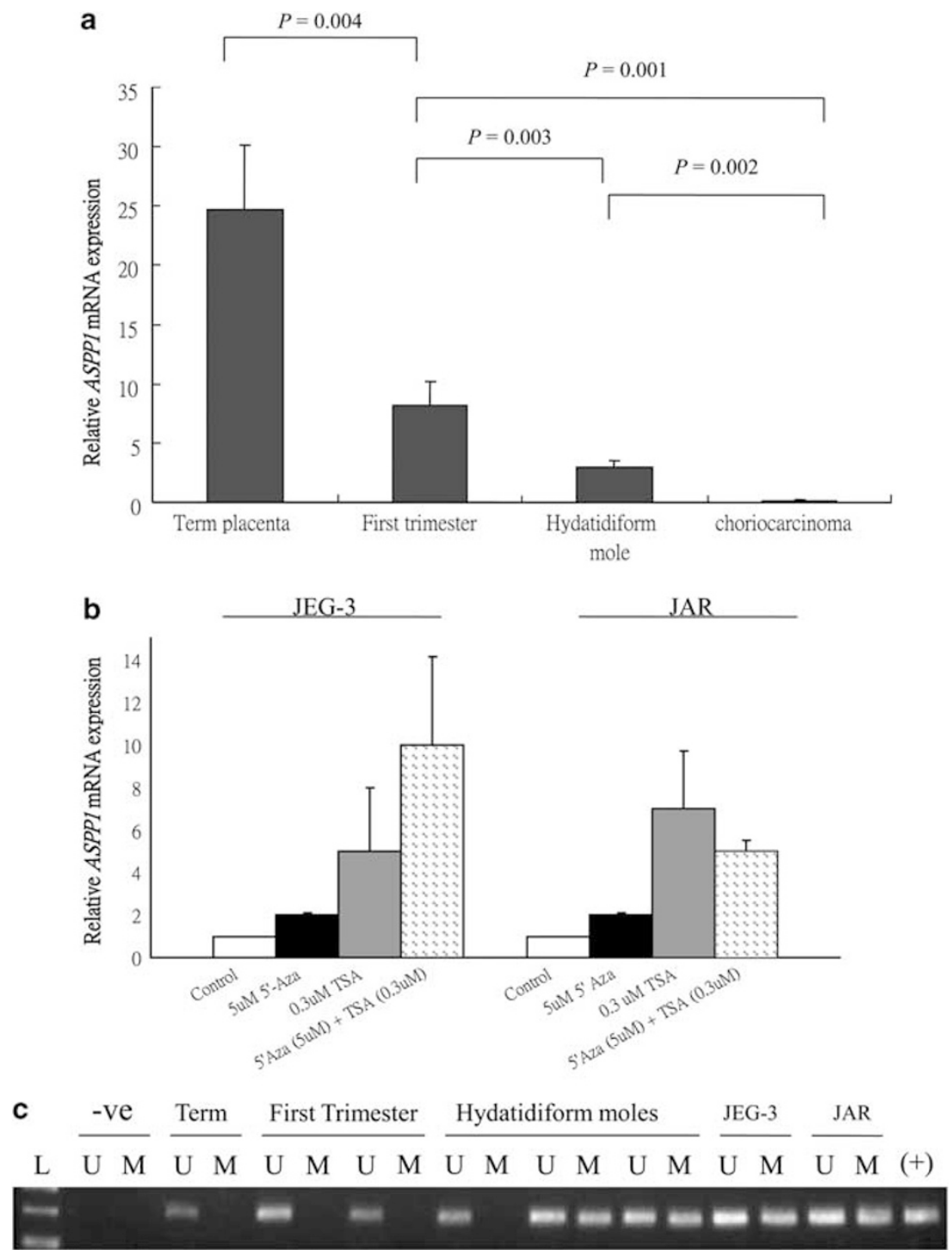

Figure 2 Studies on ASPP1 mRNA expression and methylation status in clinical samples of normal placentas and gestational trophoblastic disease. (a) $A S P P 1$ mRNA was significantly reduced in hydatidiform moles $(P=0.003)$ and choriocarcinomas $(P=0.001)$ when compared with first-trimester placentas. Term placentas showed higher mRNA level than did first-trimester placentas $(P=0.004)$. (b) Relative ASPP1 mRNA expression after 5-aza-2'-deoxycytidine (5-ada-dc) and trichostatin A (TSA) treatment. After 5-aza-dc (5 $\mu \mathrm{M})$ treatment, ASPP1 expression was upregulated by twofold in JEG-3 and JAR. Fivefold and sevenfold changes were detected in JEG-3 and JAR, respectively, after $(0.3 \mu \mathrm{M})$ TSA treatment. Increased ASPP1 mRNA expressions were also observed in treatments of both drugs. (c) Representative photograph of MS-PCR results on ASPP1 promoter methylation in normal placentas and gestational trophoblastic disease. Unmethylated (U) alleles could be observed in all samples. Methylated (M) alleles were detected in 11.8, 22.2 and $34.8 \%$ of first-trimester placentas, term placentas (Term) and hydatidiform mole samples, respectively. Both methylated and unmethylated alleles were demonstrated in choriocarcinoma cell lines. + ve: universal methylated DNA control; -ve: no template control; L: DNA marker.

Figure 1 Immunohistochemical profile of ASPP1 in trophoblastic tissues. (a-e) Immunoreactivity of ASPP1 in the first-trimester placenta (panel a), regressed mole (panel b), progressed mole (panel c) and choriocarcinoma (panel d) was mainly detected in the cytoplasm of cytotrophoblast (CT) and villous intermediate trophoblast (VIT) as indicated by arrows. Asterisk $\left({ }^{*}\right)$ indicates the location of cistern. In term placenta (panel e) where the populations of CT and VIT are inconspicuous, ASPP1 expression was noted in the syncytiotrophoblast. (f) Bar charts of immunohistochemical scores (IHS) of ASPP1 in the term placenta (term), first-trimester placenta (1st Tri), regressed mole (HM-Regressed), progressed mole (HM-Progressed) and choriocarcinomas (CCA) in trophoblasts. (g) Subcellular localization of ASPP1 expression in JEG-3 cells by western blot. 
cells of 2.4 and $3.5 \%$, respectively, whereas overexpression of ASPP1 increased the proportion of apoptotic cells to 9.4 and $13.3 \%$, respectively
(Figure 3). In addition, ASPP1-transfected choriocarcinoma cell lines showed an increase in cleaved caspase-9, indicating that ASPP1 could stimulate a

Control

ASPP1

transfected

b

\section{Control}
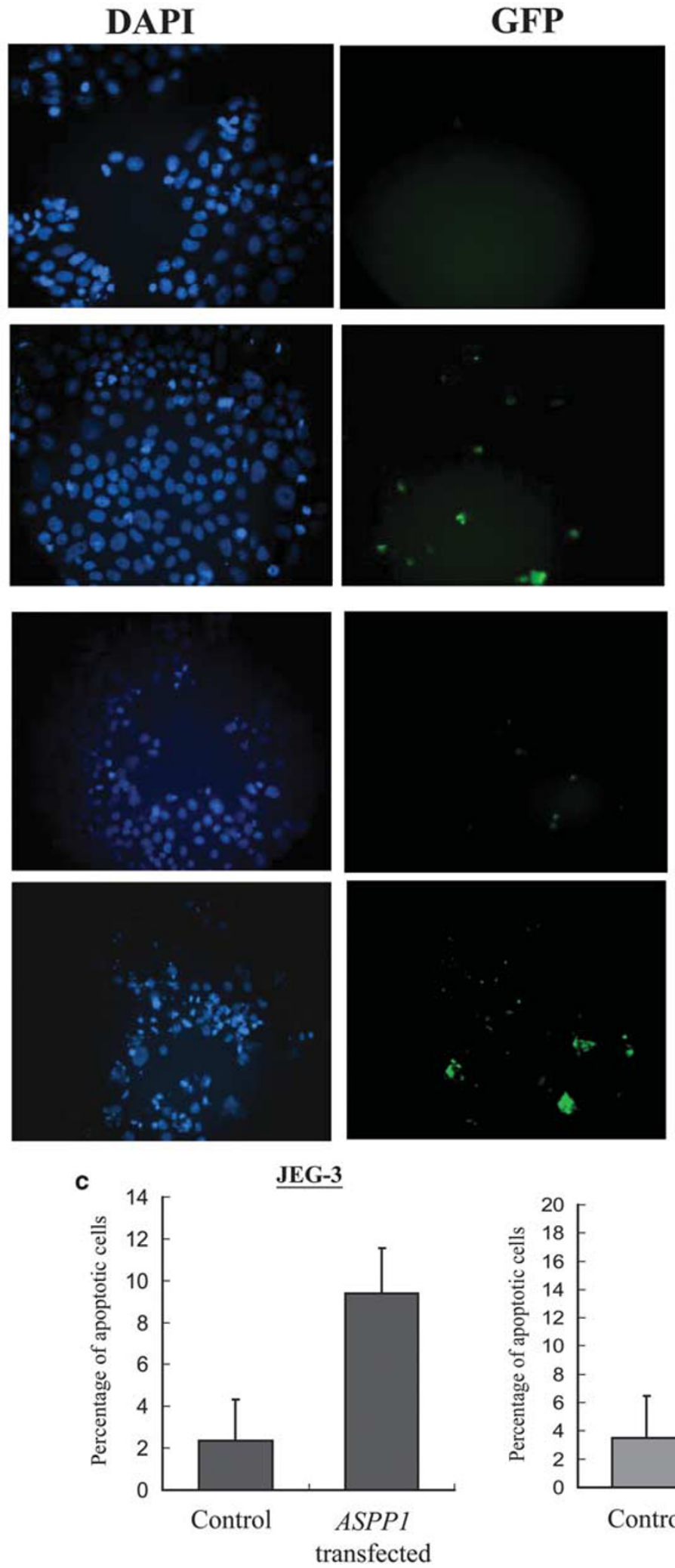

\section{ASPP1} transfected
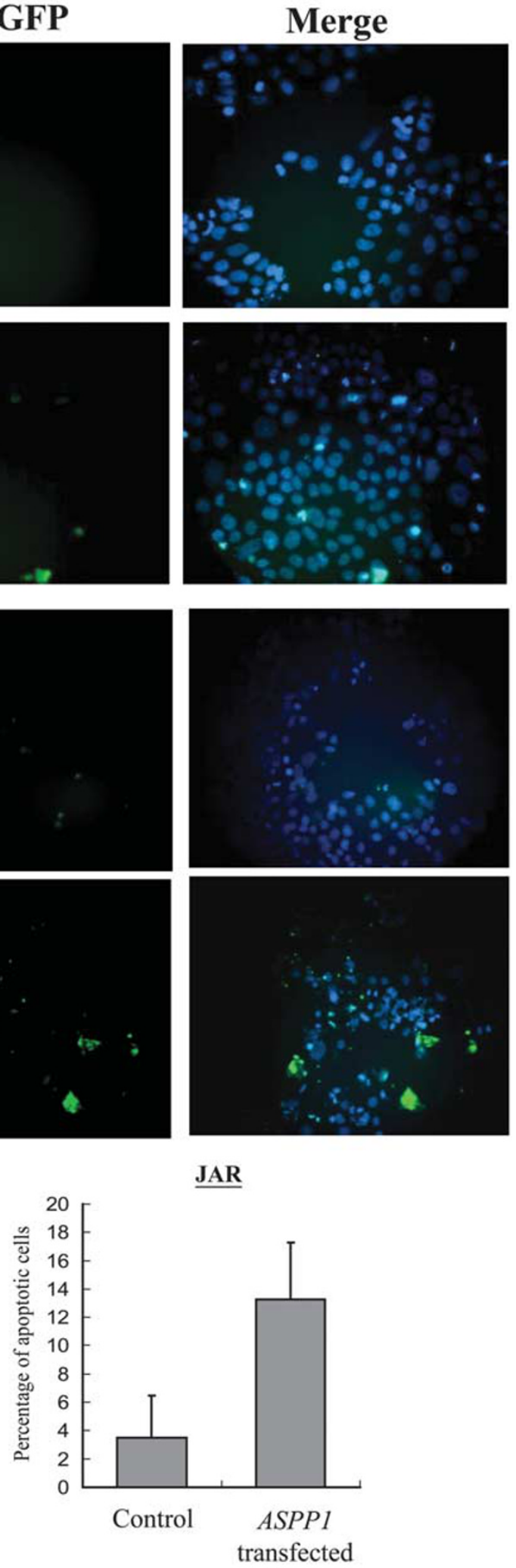

Figure 3 Apoptosis in ASPP1-transfected choriocarcinoma cells. Photographs of representative fields in (a) JEG-3 and (b) JAR in TUNEL assay. Lower panel (c) showed the percentage of apoptotic cells (apoptotic cells/total cells counted) increased after ASPP1 transfection. 


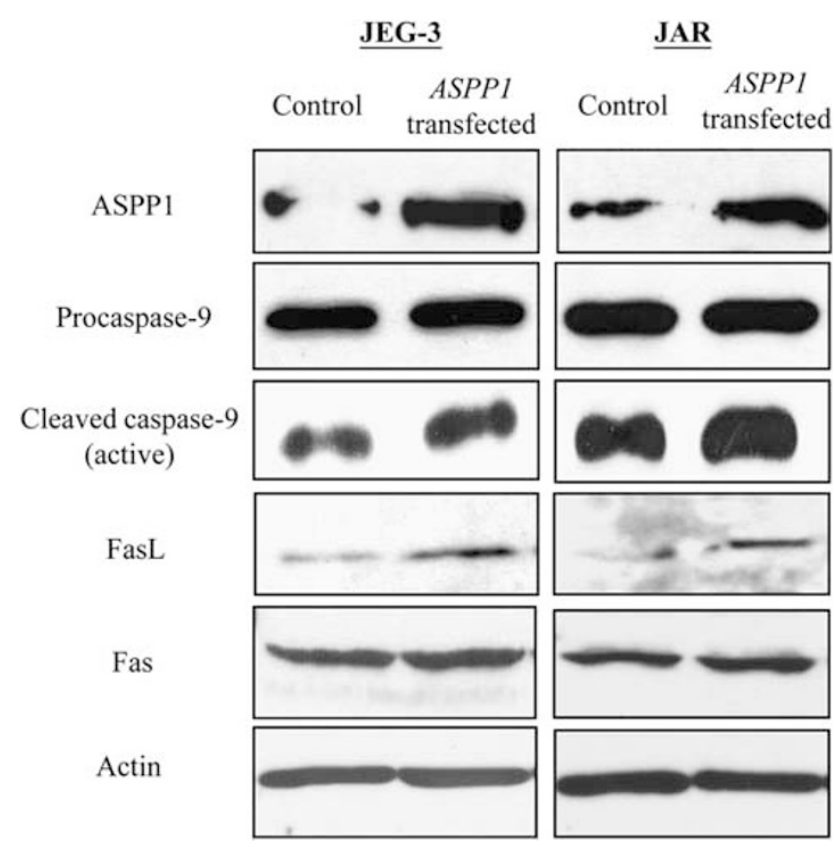

Figure 4 Western blot analysis. Western blot analysis showed increase in the expression of cleaved caspase-9 and FasL after ASPP1 overexpression. Actin was used as the loading control.

apoptosis through the intrinsic pathway of apoptosis $^{11,12}$ (Figure 4).

On the other hand, we also demonstrated a positive correlation between immunohistoscore of ASPP1 and caspase-8, which is an effector molecule in the extrinsic pathway of apoptosis that has been shown to be downregulated in gestational trophoblastic disease. ${ }^{19}$ By qPCR study following ASPP1 ectopic overexpression, increased mRNA expression of the Fas ligand (FasL) was found, but not that of TRADD and TRAF2. A modest increase in mRNA expression of Fas, a receptor for FasL, was also observed. Subsequent western blot analysis confirmed the upregulation of FasL at the protein level after ASPP1 overexpression (Figures 4 and 5). Together with the parallel downregulation of ASPP1 and caspase-8 in gestational trophoblastic disease, ${ }^{19}$ our data suggested for the first time that ASPP1 might also operate the extrinsic pathway of apoptosis, probably through the Fas/FasL system.

\section{Discussion}

\section{Aberrant Expression and Hypermethylation of ASPP1} in Gestational Trophoblastic Disease

Our immunohistochemical and fractional western blot studies on trophoblastic samples and cell lines demonstrated that ASPP1 immunoreactivity was mainly located in the cytoplasm of cytotrophoblasts and villous intermediate trophoblasts, a finding concurring with the original report when ASPP1 was first identified. ${ }^{11,27}$ In this study, the ASPP1

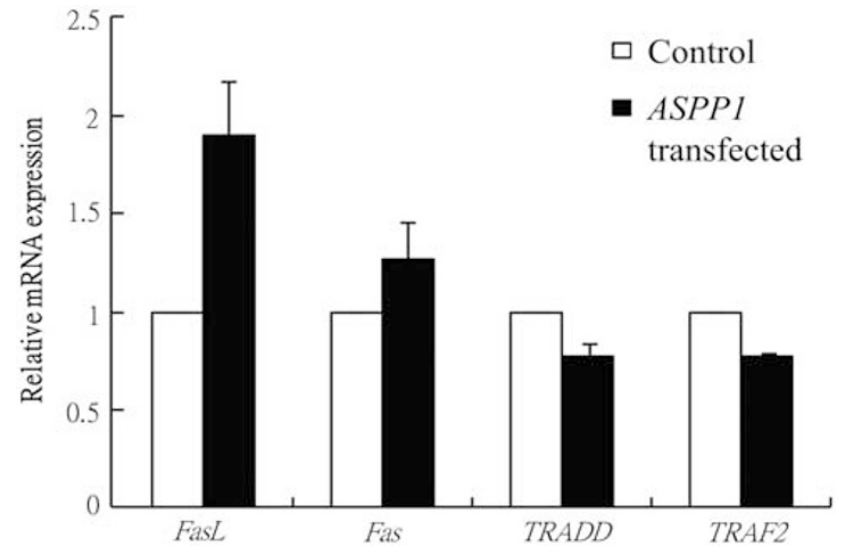

Figure 5 Upregulated FasL expression at the mRNA level. A significant relative increase in mRNA expression of the Fas ligand (FasL) $(P=0.037)$ and to a mild extent, that of Fas, but not of TRADD and TRAF2, were demonstrated in ASPP1-transfected JEG-3 cells.

antibody LOX54.2 was used and it recognized the $\mathrm{N}$ terminus (1-308) of ASPP1. Full-length ASPPs with an intact $\mathrm{N}$-terminal regulatory region are required for maximal activity. Transfection of ASPP1 lacking the N-terminal region results in less stimulation of transactivation of p53 than its full-length counterpart. ${ }^{11}$ Hence, the anti-ASPP1 antibody LOX54.2 can recognize endogenous full-length ASPP1 exhibiting complete p53 regulatory function.

Although p53 expression is located in the nucleus and ASPP1 expression is found in the cytoplasm, it has been suggested that ASPP1 contains a targeting sequence that directs it to the nucleus. Transfection of the central ASPP1 region (872-1090) and green fluorescent protein (GFP) fusion protein resulted in predominant nuclear localization of the construct, whereas full-length ASPP1 (1-1090)-GFP fusion protein was strongly cytoplasmic; the latter agreed with our immunohistochemical results. ${ }^{28}$ In this regard, it is possible that post-translational modification or binding partner is involved in the aid of the trafficking of ASPP1 in different subcellular compartments to fully execute its biological functions. On the other hand, the localization of ASPP1 expression to cytotrophoblast and villous intermediate trophoblast is intriguing as the gestational trophoblastic neoplasm is likely to be derived from these cells related to their stem cell properties and progenitor characteristics. ${ }^{29}$

Our methylation studies involving MS-PCR analysis and 5-aza-dc treatment in relation to mRNA levels support the contribution of aberrant DNA methylation to ASPP1 downregulation during the development of gestational trophoblastic disease. DNA hypermethylation of tumor-suppressor genes and stem cell transcription factors has indeed been reported in gestational trophoblastic disease. ${ }^{24,26,30}$ Moreover, synergistic reactivation of ASPP1 mRNA expression could be observed in combined 
treatment by the histone deacetylase (HDAC) inhibitor TSA and the demethylating agent 5-aza-dc, as in our previous studies on Oct4 and Sox $2 .{ }^{24,30}$ Genomic DNA methylation and histone deacetylation are essential but not secluded epigeneticregulating mechanisms. ${ }^{31}$ Methylcytosine-binding proteins, which bind to methylated DNA sequences, can recruit HDAC and histone methyltransferases, ${ }^{32}$ whereas DNA methyltransferases can directly bind HDAC, representing an interaction between DNA methylation and histone deacetylation. ${ }^{32,33}$ Interestingly, TSA alone could result in a more dramatic induction of ASPP1 mRNA expression than 5-aza-dc. Recent studies have discovered that TSA can induce DNA demethylation in some methylated tumorsuppressor genes because of its effect on histone acetylation. $^{34,35}$ ASPP1 expression can thus be affected by this selective cross-action of TSA on histone acetylation and DNA demethylation.

\section{Relationship Between ASPP1, p53, Proliferative and Apoptotic Index in the Neoplastic Transformation of Gestational Trophoblastic Disease}

Overexpression of wild-type p53 $3^{6-8,20,21}$ and higher apoptotic activities ${ }^{3,4,9,36}$ have been reported in hydatidiform moles and choriocarcinomas than in normal or hydropic placentas. It has been suggested that overexpression of wild-type p53 accompanying increased apoptotic activity would be a protective mechanism to counterbalance the uncontrolled proliferation in gestational trophoblastic disease. ${ }^{2}$ On the other hand, an inverse correlation was found between ASPP1 expression and p53 immunoreactivity and proliferative activity (Ki67 and MCM7 indices), as well as apoptotic activity (M30 index). These inverse correlations suggested that ASPP1 downregulation may be important in the balance between proliferation and apoptotic activities and may contribute to the development of gestational trophoblastic disease.

We have demonstrated earlier that hydatidiform moles that developed gestational trophoblastic neoplasms have significantly lower apoptotic index than do those that were spontaneously regressed $d^{3,4}$ in association with dyregulation of apoptosisrelated genes. ${ }^{22,23,37}$ In this study, we found an inverse correlation between ASPP1 expression and clinical progress of hydatidiform moles. It is possible that the progressive loss of proapoptotic ASPP1 expression hinders the usual enhanced apoptosis in cytotrophoblasts and villous intermediate trophoblasts, leading to the development of persistent gestational trophoblastic neoplasms.

\section{ASPP1 and Apoptotic Cascades in Gestational Trophoblastic Disease}

Through the binding to p53, ASPP1 can transactivate the transcription of proapoptotic genes such as $B A X$ and $P U M A$ in the intrinsic pathway. ${ }^{11,12}$ Our results showed that induced expression of ASPP1 in choriocarcinoma cell lines which retain wild-type p53 ${ }^{38}$ could trigger apoptosis, in association with increased expression of the activated (cleaved) form of caspase-9 confirming the activation of the intrinsic pathway by ASPP1.

On the other hand, we also found an association between ASPP1 and total caspase- 8 expression (proform and cleaved form). Caspase-8, a member of the extrinsic pathway of apoptosis, has indeed been identified in our previous apoptosis array study to be significantly downregulated in hydatidiform moles when compared with normal placentas. ${ }^{19}$ We demonstrate for the first time the involvement of ASPP1 in the extrinsic pathway of apoptosis by confirming the coexpression Fas and FasL in choriocarcinoma cell lines and the upregulation of FasL subsequent to ASPP1 transfection. Coexpression of Fas and FasL is implicated in apoptotic cell death during physiological cell turnover of tissues, ${ }^{39}$ and FasL and Fas can interact by paracrine and autocrine mechanisms in trophoblasts. ${ }^{40}$ In brief, our results implied for the first time, the possible interaction between ASPP1 and Fas/FasL system and thus the extrinsic pathway of apoptosis especially in the germinating cytotrophoblast.

In summary, we have demonstrated the downregulation of proapoptotic $A S P P 1$ by hypermethylation in gestational trophoblastic disease in association with aggressive outcome of hydatidiform mole. We also report for the first time a potential interaction of ASPP1 with the Fas/FasL system of the extrinsic pathway of apoptosis in trophoblasts. It is possible that ASPP1 through its effect on apoptotic activity, may have important roles in the pathogenesis of gestational trophoblastic disease.

\section{Acknowledgement}

This study was supported by grants from the 'Centre for Research into Circulating Fetal Nucleic Acids' (AoE/M-04/06) funded by the Research Grants Council of the Hong Kong Special Administrative Region.

\section{Disclosure/conflict of interest}

The authors declare no conflict of interest.

\section{References}

1 Altieri A, Franceschi S, Ferlay J, et al. Epidemiology and aetiology of gestational trophoblastic diseases. Lancet Oncol 2003;4:670-678.

2 Li HW, Tsao SW, Cheung AN. Current understandings of the molecular genetics of gestational trophoblastic diseases. Placenta 2002;23:20-31. 
3 Chiu PM, Ngan YS, Khoo US, et al. Apoptotic activity in gestational trophoblastic disease correlates with clinical outcome: assessment by the caspase-related M30 CytoDeath antibody. Histopathology 2001;38: 243-249.

4 Wong SY, Ngan HY, Chan CC, et al. Apoptosis in gestational trophoblastic disease is correlated with clinical outcome and Bcl-2 expression but not Bax expression. Mod Pathol 1999;12:1025-1033.

5 Shaw P, Bovey R, Tardy S, et al. Induction of apoptosis by wild-type p53 in a human colon tumor-derived cell line. Proc Natl Acad Sci USA 1992;89:4495-4499.

6 Cheung AN, Srivastava G, Chung LP, et al. Expression of the p53 gene in trophoblastic cells in hydatidiform moles and normal human placentas. J Reprod Med 1994;39:223-227.

7 Lee YS. p53 expression in gestational trophoblastic disease. Int J Gynecol Pathol 1995;14:119-124.

8 Fulop V, Mok SC, Genest DR, et al. p53, p21, Rb and mdm2 oncoproteins. Expression in normal placenta, partial and complete mole, and choriocarcinoma. J Reprod Med 1998;43:119-127.

9 Halperin R, Peller S, Sandbank J, et al. Expression of the p53 gene and apoptosis in gestational trophoblastic disease. Placenta 2000;21:58-62.

10 Shi YF, Xie X, Zhao CL, et al. Lack of mutation in tumour-suppressor gene p53 in gestational trophoblastic tumours. Br J Cancer 1996;73:1216-1219.

11 Samuels-Lev Y, O’Connor DJ, Bergamaschi D, et al. ASPP proteins specifically stimulate the apoptotic function of p53. Mol Cell 2001;8:781-794.

12 Bergamaschi D, Samuels Y, Jin B, et al. ASPP1 and ASPP2: common activators of p53 family members. Mol Cell Biol 2004;24:1341-1350.

13 Bergamaschi D, Samuels Y, O’Neil NJ, et al. iASPP oncoprotein is a key inhibitor of p53 conserved from worm to human. Nat Genet 2003;33:162-167.

14 Liu ZJ, Zhang Y, Zhang XB, et al. Abnormal mRNA expression of ASPP members in leukemia cell lines. Leukemia 2004;18:880.

15 Mori S, Ito G, Usami N, et al. p53 apoptotic pathway molecules are frequently and simultaneously altered in nonsmall cell lung carcinoma. Cancer 2004;100: 1673-1682.

16 Agirre X, Roman-Gomez J, Jimenez-Velasco A, et al. ASPP1, a common activator of TP53, is inactivated by aberrant methylation of its promoter in acute lymphoblastic leukemia. Oncogene 2006;25: 1862-1870.

17 Lai CY, Chan KY, Khoo US, et al. Analysis of gestational trophoblastic disease by genotyping and chromosome in situ hybridization. Mod Pathol 2004; 17:40-48.

18 Cheung AN, Khoo US, Lai CY, et al. Metastatic trophoblastic disease after an initial diagnosis of partial hydatidiform mole: genotyping and chromosome in situ hybridization analysis. Cancer 2004;100: 1411-1417.

19 Fong PY, Xue WC, Ngan HY, et al. Caspase activity is downregulated in choriocarcinoma: a cDNA array differential expression study. J Clin Pathol 2006;59: 179-183.

20 Xue WC, Khoo US, Ngan HY, et al. Minichromosome maintenance protein 7 expression in gestational trophoblastic disease: correlation with Ki67, PCNA and clinicopathological parameters. Histopathology 2003;43:485-490.
21 Cheung AN, Shen DH, Khoo US, et al. Immunohistochemical and mutational analysis of p53 tumor suppressor gene in gestational trophoblastic disease: correlation with mdm2, proliferation index, and clinicopathologic parameters. Int J Gynecol Cancer 1999;9:123-130.

22 Chan HY, Siu MK, Zhang HJ, et al. Activated Stat3 expression in gestational trophoblastic disease: correlation with clinicopathological parameters and apoptotic indices. Histopathology 2008;53:139-146.

23 Siu MK, Wong ES, Chan HY, et al. Overexpression of NANOG in gestational trophoblastic diseases: effect on apoptosis, cell invasion, and clinical outcome. Am J Pathol 2008;173:1165-1172.

24 Zhang HJ, Siu MK, Wong ES, et al. Oct4 is epigenetically regulated by methylation in normal placenta and gestational trophoblastic disease. Placenta 2008;29: $549-554$.

25 Xue WC, Feng HC, Tsao SW, et al. Methylation status and expression of E-cadherin and cadherin-11 in gestational trophoblastic diseases. Int J Gynecol Cancer 2003;13:879-888.

26 Xue WC, Chan KY, Feng HC, et al. Promoter hypermethylation of multiple genes in hydatidiform mole and choriocarcinoma. J Mol Diagn 2004;6: 326-334.

27 Bergamaschi D, Samuels Y, Zhong S, et al. Mdm2 and mdmX prevent ASPP1 and ASPP2 from stimulating p53 without targeting p53 for degradation. Oncogene 2005;24:3836-3841.

28 Thornton JK, Dalgleish C, Venables JP, et al. The tumour-suppressor protein ASPP1 is nuclear in human germ cells and can modulate ratios of CD44 exon V5 spliced isoforms in vivo. Oncogene 2006;25: 3104-3112.

29 Shih Ie M. Gestational trophoblastic neoplasiapathogenesis and potential therapeutic targets. Lancet Oncol 2007;8:642-650.

30 Li AS, Siu MK, Zhang H, et al. Hypermethylation of SOX2 gene in hydatidiform mole and choriocarcinoma. Reprod Sci 2008;15:735-744.

31 Fahrner JA, Eguchi S, Herman JG, et al. Dependence of histone modifications and gene expression on DNA hypermethylation in cancer. Cancer Res 2002;62: 7213-7218.

32 Fuks F, Burgers WA, Brehm A, et al. DNA methyltransferase Dnmt1 associates with histone deacetylase activity. Nat Genet 2000;24:88-91.

33 Rountree MR, Bachman KE, Baylin SB. DNMT1 binds HDAC2 and a new co-repressor, DMAP1, to form a complex at replication foci. Nat Genet 2000;25: 269-277.

$34 \mathrm{Ou}$ JN, Torrisani J, Unterberger A, et al. Histone deacetylase inhibitor Trichostatin A induces global and gene-specific DNA demethylation in human cancer cell lines. Biochem Pharmacol 2007;73: 1297-1307.

35 Cervoni N, Szyf M. Demethylase activity is directed by histone acetylation. J Biol Chem 2001;276: 40778-40787.

36 Kim MJ, Kim KR, Ro JY, et al. Diagnostic and pathogenetic significance of increased stromal apoptosis and incomplete vasculogenesis in complete hydatidiform moles in very early pregnancy periods. Am J Surg Pathol 2006;30:362-369.

37 Fong PY, Xue WC, Ngan HY, et al. Mcl-1 expression in gestational trophoblastic disease correlates with clin- 
ical outcome: a differential expression study. Cancer 2005;103:268-276.

38 Chen CA, Chen YH, Chen TM, et al. Infrequent mutation in tumor suppressor gene p53 in gestational trophoblastic neoplasia. Carcinogenesis 1994;15: 2221-2223.

39 French LE, Hahne M, Viard I, et al. Fas and Fas ligand in embryos and adult mice: ligand expression in several immune-privileged tissues and coexpression in adult tissues characterized by apoptotic cell turnover. J Cell Biol 1996;133: 335-343.

40 Balkundi DR, Ziegler JA, Watchko JF, et al. Regulation of FasL/Fas in human trophoblasts: possible implications for chorioamnionitis. Biol Reprod 2003;69: 718-724. 